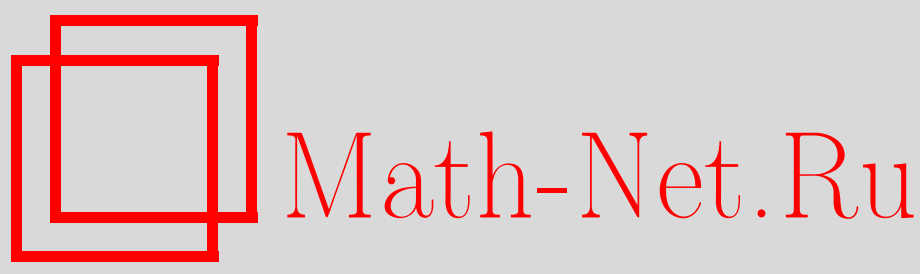

А. М. Стоколос, О дифференцировании интегралов базисами, не обладающими свойством плотности, Матем. сб., 1996, том 187, номер 7, 113-138

DOI: https://doi.org/10.4213/sm148

Использование Общероссийского математического портала Math-Net.Ru подразумевает, что вы прочитали и согласны с пользовательским соглашением

http://www . mathnet.ru/rus/agreement

Параметры загрузки:

IP: 54.197 .217 .227

26 апреля 2023 г., 10:14:59 
УДК 517.5

\author{
А.М. Стоколос
}

\title{
О дифференцировании интегралов базисами, не обладающими свойством плотности
}

\footnotetext{
В работе рассматриваются вопросы дифференцирования интегралов функций из классов Гёльдера некоторыми достаточно общими базисами, включая базис из вьпуклых множеств.

Библиографияя: 23 названия.
}

\section{§1. Введение}

Напомним вначале основные определения (см. также [1], [2]).

Пусть $\mathscr{B}$ - дифференциальный базис. Говорят, что $\mathscr{B}$ дифференцирует интез$p a \Omega$ от суммируемой функции $f$, если для почти всех $x$ из области определения $f$ вьполняется соотношение

$$
\lim _{\operatorname{diam}(R) \rightarrow 0} \frac{1}{|R|} \int_{\mathscr{B} \ni R \ni x} f(y) d y=f(x) .
$$

Если базис $\mathscr{B}$ дифференцирует интегралы всех функций из некоторого класса $\mathscr{K}$, то говорят, что $\mathscr{B}$ дифференцирует $\mathscr{K}$. В случае, когда базис дифференцирует характеристические функции измеримых множеств, его называют nлотностным, или обладающим свойством плотности.

Г. Буземанн и В. Феллер [3] показали, что свойство плотности эквивалентно дифференцированию $L^{\infty}$. Вместе с тем, известные в настоящее время инвариантные относительно сдвига плотностные базисы дифференцируют $L^{p}, p>1$. Вполне возможно, что это является общей закономерностью для такого типа базисов.

Перечислим основные плотностные базисы. Это - базис из многомерных шаров (А. Лебег, 1910, [4]), многомерных интервалов (С. Сакс, 1933, [5]), прямоугольников на плоскости, ориентированных в лакунарных направлениях (Я. О. Стрёмберг, $1977,[6])$ и прямоугольников на плоскости в итерированных лакунарных направлениях (П. Шёгрен, П. Шёлин, 1987, [7]).

Базис из произвольно ориентированных прямоугольников не является плотностным. Этот факт установил А. Зигмунд в 1927 году, изучая принадлежащую О. Никодиму конструкцию одного парадоксального множества [8].

Приведенные вьше факты лежат в основе теории дифференцирования интегралов, имеющей дело либо с классами $L^{p}, 1 \leqslant p \leqslant \infty$, либо с классами Орлича. При этом, естественно, рассматриваются плотностные базисы.

Работа выполнена при частичной финансовой поддержке объединенного Фонда Правительства Украины и Международного научного фонда (грант № K 41100). 
Базисы, не обладающие свойством плотности, в силу отсутствия соответству-

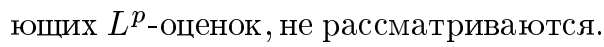

В настоящей работе предложен подход к изучению неплотностных базисов, основанный на замене $L^{p}$-оценок $H_{p}^{\omega}$-оценками. В результате, получен ряд утверждений, некоторые из которых носят окончательный, в своих терминах, характер.

В 33 мы рассматриваем вопросы дифференцирования интегралов базисами самого общего вида и показываем, что в общем случае условия дифференцирования не отличаются от условия вложения в класс непрерьвных функций, где дифференцируемость тривиальна.

В $₫ 4$ мы вводим базис достаточно обшего вида, который естественным образом определяется по результатам предыдущего параграфа. Для этого базиса устанавливаются некоторые положительные результаты. При этом, как это часто бывает в теории вложения функциональных пространств, случаи $p=1$ и $p>1$ рассматриваются в рамках разных схем рассуждений.

В $\S 5$ изучается окончательность полученных вьше результатов. При этом выясняется, что в случае $p=1$ аналогичный результат справедлив и для базиса из вьпуклых множеств, который можно считать наиболее интересным и естественным в рассматриваемой тематике. В случае же $p>1$ нам не удалось получить соответствуюшую оценку снизу для базиса из вьпуклых множеств. Более того, в случае $1<p<2$ получена оценка снизу степенного типа (как и оценка сверху), а в случае $p \geqslant 2$ оценка снизу и вовсе носит логарифмический характер. При этом, мы даже затрудняемся высказать предположение о том, какой же все-таки порядок является правильным.

В заключение автор выражает благодарность Ю.В. Крякину за полезные обсуждения и оказанную помощь при подготовке статьи.

\section{§ 2. Некоторые обозначения}

Символами « и 》 мы будем обозначать неравенства $\leqslant$ и $\geqslant$ с некоторой положительной константой в правой части.

Всякую возрастаюшую на $(0,1]$ полуаддитивную функцию $\omega(\delta), \omega(0+)=0$, будем назьвать модулем непрерьвности.

Пусть $d \geqslant 2 ; 1 \leqslant j \leqslant d ; h \in(0,1] ; I_{h, j}^{d}=\left\{x \in I^{d}: 0 \leqslant x_{j} \leqslant 1-h\right\}$. Для $f \in L^{p}\left(I^{d}\right), p \geqslant 1$, введем $j$-й частный модуль непрерывности

$$
\omega_{j}(f ; \delta)_{p}=\sup _{0<h \leqslant \delta}\left\{\int_{I_{h, j}^{d}}\left|f\left(x_{1}, \ldots, x_{j}+h, \ldots, x_{d}\right)-f\left(x_{1}, \ldots, x_{j}, \ldots, x_{d}\right)\right|^{p}\right\}^{1 / p} .
$$

Для 1-периодических по каждой переменной функций $f \in L^{p}\left(I^{d}\right), p \geqslant 1$, определим периодический $j$-ый частный модуль непрерывности

$$
\widetilde{\omega}_{j}(f ; \delta)_{p}=\sup _{0<h \leqslant \delta}\left\{\int_{I^{d}}\left|f\left(x_{1}, \ldots, x_{j}+h, \ldots, x_{d}\right)-f\left(x_{1}, \ldots, x_{j}, \ldots, x_{d}\right)\right|^{p}\right\}^{1 / p} .
$$

Определим теперь изотропный модуль непрерывности функции $f$

$$
\omega(f ; \delta)_{p} \equiv \max _{i=1, \ldots, d} \omega_{i}(f ; \delta)_{p} .
$$


Периодический изотропньй модуль непрерывности задается таким же соотношением, с заменой $\omega_{i}(f ; \delta)_{p}$ на $\widetilde{\omega}_{i}(f ; \delta)_{p}$.

Введем в рассмотрение классы Гёльдера

$$
H_{p}^{\omega}\left(I^{d}\right)=\left\{f \in L^{p}\left(I^{d}\right): \omega(f ; \delta)_{p}=O(\omega(\delta))\right\} .
$$

Аналогичным образом определяются периодические классы Гёльдера $\widetilde{H}_{p}^{\omega}\left(I^{d}\right)$.

Через $B_{p, q}^{s}\left(I^{d}\right)$ обозначим пространство Бесова с нормой

$$
\|f\|_{B_{p, q}^{s}}=\|f\|_{p}+\left\{\int_{0}^{1}\left(\frac{\omega(f ; \delta)_{p}}{\delta^{s}}\right)^{q} \frac{d \delta}{\delta}\right\}^{1 / q} .
$$

\section{§ 3. Базисы общего вида}

Условия, при которых справедливы вложения $H_{p}^{\omega}\left(I^{d}\right) \subset C\left(I^{d}\right)$ и $\widetilde{H}_{p}^{\omega}\left(I^{d}\right) \subset$ $C\left(I^{d}\right)$ исследовались в работах П. Л. Ульянова [9], Н. Темиргалиева [10], В.И. Коляды [11]. Выяснилось, что при $p>1$ следуюшее условие является необходимым и достаточным для указанного вложения:

$$
\int_{0}^{1} \frac{\omega(\delta)}{\delta^{d / p+1}} d \delta<\infty
$$

Таким образом, если $\omega(\delta)$ удовлетворяет условию $(2)$, то классы $H_{p}^{\omega}\left(I^{d}\right)$ и $\widetilde{H}_{p}^{\omega}\left(I^{d}\right)$ будут дифференцироваться любым базисом. В обшем случае ожидать ослабления (2) не приходится, что действительно так.

Введем в рассмотрение базис $\mathscr{B}_{0}$, состояший из множеств $E$, содержащих шар $Q$, таких, что $|E| \leqslant 2|Q|$ (см. рис. 1 ).

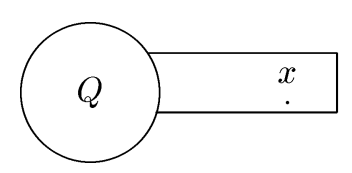

Рис. 1

Пусть теперь (2) не выполнено. Покажем, что тогда базис $\mathscr{B}_{0}$ не дифференцирует $\widetilde{H}_{p}^{\omega}\left(I^{d}\right)$.

В этом случае существует 1-периодическая по каждой переменной существенно неограниченная функция $f \in \widetilde{H}_{p}^{\omega}\left(I^{d}\right)$. Поскольку модуль функции $f$ также из $\widetilde{H}_{p}^{\omega}\left(I^{d}\right)$, то можно считать $f \geqslant 0$.

Занумеруем рациональные точки из $I^{d}$. Сдвинув $f$ в $k$-ю рациональную точку из $I^{d}$, результат сдвига обозначим $f_{k}$. В силу периодичности $\widetilde{\omega}_{j}\left(f_{k} ; \delta\right)_{p}=$ $\widetilde{\omega}_{j}(f ; \delta)_{p}$, следовательно, функция

$$
F=\sum_{k=1}^{\infty} f_{k} 2^{-k}
$$


также принадлежит классу $\widetilde{H}_{p}^{\omega}\left(I^{d}\right)$.

Далее, легко видеть, что в любой окрестности произвольной точки $x$ из $I^{d}$ найдется шар $Q$, для которого средние $|Q|^{-1} \int_{Q} F$ достаточно велики. Соединив теперь точку $x$ и шар $Q$ областью $G$ так, чтобы $|G| \leqslant|Q|$, для $E=Q \cup G$ получим

$$
|E|^{-1} \int_{E \ni x} F \geqslant(2|Q|)^{-1} \int_{Q} F
$$

следовательно, интеграл от функции $F$ не дифференцируется базисом $\mathscr{B}_{0}$.

В случае же $p=1, \operatorname{Lip}(1,1)\left(I^{d}\right) \not \subset C\left(I^{d}\right)$, и вместо условия $(2)$ можно рассматривать лишш тривиальное условие $\omega(f ; \delta)_{1}=o(\delta)$, поскольку в этом случае функция эквивалентна константе. При этом, аналогичными приведенньм выше рассуждениями можно показать, что о заменить на $O$ нельзя.

Итак, если не накладывать дополнительные ограничения на базисы, то только условия вложения в классы непрерывных функций обеспечивают дифференцирование классов Гёльдера.

Однако, для некоторых достаточно естественных базисов, например для базисов из выпуклых множеств, это не так.

K рассмотрению этих базисов мы и переходим.

\section{§4. Базис из гибких цилиндров}

Введенный в рассмотрение в предыдущем параграфе базис $\mathscr{B}_{0}$ имеет ту принципиальную особенность, что вертикальные сечения его множеств $E$ имеют разную меру. Мера сечения $Q$ - “главной части” множества $E$ - в среднем намного больше меры сечения остального множества, что существенно использовалось в рассуждениях. В связи с этим, нам представляется целесообразным выяснить, насколько этот момент принципиальньй.

Для этого введем следуюшие множества с равномерньми сечениями.

ОПрЕДЕлЕниЕ. Пусть $E$ - измеримое множество из $\mathbb{R}^{d}$ такое, что для некоторого $\nu \in\{1, \ldots, d\}$ проекция $E$ на $\nu$-ю координатную ось есть интервал $(a ; b)$ и для любого $t \in(a ; b)$ сечение $E$ гиперплоскостью $x_{v}=t$ (будем обозначать его $E_{t}$ ) имеет постоянную меру.

Множество $E$ назовем гибким $\nu$-иилиндром (или, просто, гибким иилиндром), а дифференциальный базис из этих множеств обозначим $\mathscr{B}_{\nu}$, и положим $\mathscr{B}=\bigcup_{\nu=1}^{d} \mathscr{B}_{\nu}$.

Очевидно,

$$
|E|=(b-a)\left|E_{t}\right| \quad \forall t \in(a ; b) .
$$

Частным случаем таких базисов является базис из многомерных интервалов. Вопросы интегральной гладкости и дифференцирования для таких базисов (так называемое “сильное дифференцирование”) рассматривались в [12].

Другим важньм частным случаем базиса из гибких цилиндров является базис из множеств на плоскости вида

$$
P(x, a, b)=\left\{\left(x_{1}+s_{1} ; x_{2}+s_{2}+s_{1}^{2}\right): s_{1} \in(0 ; a) ; s_{2} \in(0 ; b)\right\}
$$


Этот базис использовался А. Кордобой, Ч. Фефферманом и Р. Фефферманом для решения важной проблемы о дифференцировании вдоль радиального поля направлений (см. [2, с. 351]). Выбор такого базиса был ориентирован на использование теоремы Е.М. Стейна и С. Вейнгера [13], об ограниченности в $L^{p}, 1<p<\infty$, максимального оператора вдоль параболы, которая, в свою очередь, существенно учитывает алгебраическую природу параболы и для произвольных кривых места не имеет.

Обозначим через $\mathscr{M}_{\mathscr{B}} f$ максимальный оператор ассоциированный с базисом $\mathscr{B}$

$$
\mathscr{M}_{\mathscr{B}} f(x)=\sup |R|^{-1} \int_{R}|f(y)| d y,
$$

где супремум рассматривается по множеству $\{R: \mathscr{B} \ni R \ni x ; \operatorname{diam}(R)<1 / 2$; $\left.R \subset I^{d}\right\}$.

Случай $1<p<\infty$.

Лемма 1. Пусть $d \geqslant 2 ; p>d-1 ; s=(d-1) / p ; f \in B_{p, 1}^{s}\left(I^{d}\right)$. Тогда справедлива следующая оченка слабого типа

$$
\left|\left\{x \in I^{d}: \mathscr{M}_{\mathscr{B}}(x)>\lambda\right\}\right| \leqslant \frac{C(p, d)}{\lambda}\|f\|_{B_{p, 1}^{s}} \quad \forall \lambda>0 .
$$

ДокАЗАТЕльСтво. Для $g \in L^{p}\left(I^{k}\right)$ через $g^{*}(t)$ обозначим невозрастающую перестановку функции $g(x)$ (см., например, [14]). Для $0<\tau \leqslant 1 / 2$ справедливо следующее неравенство

$$
\left(\frac{1}{\tau} \int_{0}^{\tau} f^{*}(t)^{p} d t\right)^{1 / p} \leqslant 2^{1 / p}\left\{\int_{\tau}^{1 / 2} \frac{\omega\left(f ; t^{1 / k}\right)_{p}}{t^{1+1 / p}} d t+\frac{\omega\left(f ; \tau^{1 / k}\right)_{p}}{\tau^{1 / p}}+\|f\|_{p}\right\} .
$$

В случае $k=1$ это неравенство было установлено Э.А. Стороженко [15], а для $k>1$ В.И. Колядой [16].

Пусть теперь $f \in L^{p}\left(I^{d}\right)$. Через $f^{*}\left(y_{\nu}, t\right)$ обозначим невозрастающую перестановку функции $f$ по переменным $\left(y_{1}, \ldots, y_{\nu-1}, y_{\nu+1}, \ldots, y_{d}\right) \equiv \widehat{y}_{\nu}$ при фиксированном $y_{\nu}$ (см. [14]). Тогда

$$
\begin{aligned}
|E|^{-1} \int_{E}|f| & =|E|^{-1} \int_{E}\left|f\left(y_{\nu} ; \widehat{y}_{\nu}\right)\right| d y_{\nu} d \widehat{y}_{\nu} \\
& \leqslant \frac{1}{b-a} \int_{a}^{b} d y_{\nu}\left(\left|E_{y_{\nu}}\right|^{-1} \int_{0}^{\left|E_{y_{\nu}}\right|} f^{*}\left(y_{\nu} ; t\right)^{p} d t\right)^{1 / p} \\
& \ll \frac{1}{b-a} \int_{a}^{b} d y_{\nu}\left\{\int_{0}^{1 / 2} \frac{\omega\left(f\left(y_{\nu} ; \cdot\right) ; t^{1 /(d-1)}\right)_{p}}{t^{1+1 / p}} d t+\left\|f\left(y_{\nu} ; \cdot\right)\right\|_{p}\right\} d t .
\end{aligned}
$$

Отсюда, обозначив через $\mathscr{M}^{\nu} f$ максимальную функцию Харди-Литлвуда примененную по $\nu$-ой переменной, получим

$$
\mathscr{M}_{\mathscr{B}} f(x) \leqslant C_{p} \mathscr{M}^{\nu}\left\{\int_{0}^{1 / 2} \frac{\omega\left(f(\cdot ; \cdot) ; t^{1 /(d-1)}\right)_{p}}{t^{1+1 / p}} d t+\|f(\cdot ; \cdot)\|_{p}\right\}\left(x_{\nu}\right) .
$$


Поскольку $\mathscr{M}^{\nu} f$ имеет слабый тип (1-1), то справедливо следуюшее неравенстBO

$$
\begin{aligned}
& \left|\left\{x \in I^{d}: \mathscr{M}_{\mathscr{B}}(x)>\lambda\right\}\right| \\
& \quad \ll \lambda^{-1}\left[\int_{0}^{1} \int_{0}^{1 / 2} \frac{\omega\left(f\left(x_{\nu} ; \cdot\right) ; t^{1 /(d-1)}\right)_{p}}{t^{1+1 / p}} d t d x_{\nu}+\int_{0}^{1}\left\|f\left(x_{\nu} ; \cdot\right)\right\|_{p} d x_{\nu}\right] .
\end{aligned}
$$

Очевидно,

$$
\int_{0}^{1}\left\|f\left(x_{\nu} ; \cdot\right)\right\|_{p} d x_{\nu} \leqslant\|f\|_{p}
$$

и осталось оценить первое слагаемое. Для этого привлечем следуюшее соотношение $[17$, с. $83,(4.27)]$ :

$$
\omega_{j}(f ; \delta)_{p} \asymp\left\{\frac{1}{\delta} \int_{0}^{\delta} \int_{I_{h, j}^{k}}\left|\Delta_{h}^{j} f(x)\right|^{p} d x d h\right\}^{1 / p}
$$

справедливое для $1 \leqslant p<\infty, 0<\delta \leqslant 1 / 2, f \in L^{p}\left(I^{k}\right)$. С учетом этого соотношения и неравенства Йенсена получим

$$
\begin{aligned}
\int_{0}^{1} & \int_{0}^{1 / 2} \frac{\omega\left(f\left(x_{\nu} ; \cdot\right) ; t^{1 /(d-1)}\right)_{p}}{t^{1+1 / p}} d t d x_{\nu} \\
& \ll \sum_{j=1, j \neq \nu}^{d} \int_{0}^{1 / 2} \frac{d t}{t^{1+(d-1) / p}} \int_{0}^{1} d x_{\nu}\left\{\frac{1}{t} \int_{0}^{t} \int_{I_{h, j}^{d-1}}\left|\Delta_{h}^{j} f\left(\widehat{x}_{\nu}\right)\right|^{p} d \widehat{x}_{\nu} d h\right\}^{1 / p} \\
& \ll \sum_{j=1, j \neq \nu}^{d} \int_{0}^{1 / 2} \frac{d t}{t^{1+(d-1) / p}}\left\{\frac{1}{t} \int_{0}^{t} \int_{I_{h, j}^{d}}\left|\Delta_{h}^{j} f(x)\right|^{p} d x d h\right\}^{1 / p} \\
& \ll \sum_{j=1}^{d} \int_{0}^{1 / 2} \frac{\omega_{j}(f ; t)_{p}}{t^{1+(d-1) / p}} d t \ll \int_{0}^{1} \frac{\omega(f ; t)_{p}}{t^{1+(d-1) / p}} d t
\end{aligned}
$$

что и дает оценку требуемого типа. Лемма доказана.

С помощью этой леммы докажем следующую теорему.

ТЕОРема 1. Для того чтобъ базис $\mathscr{B}$ дифференцировал $H_{p}^{\omega}\left(I^{d}\right), p>1$, необходимо и достаточно, чтобы

$$
\int_{0}^{1} \frac{\omega(t) d t}{t^{1+(d-1) / p}}<\infty
$$




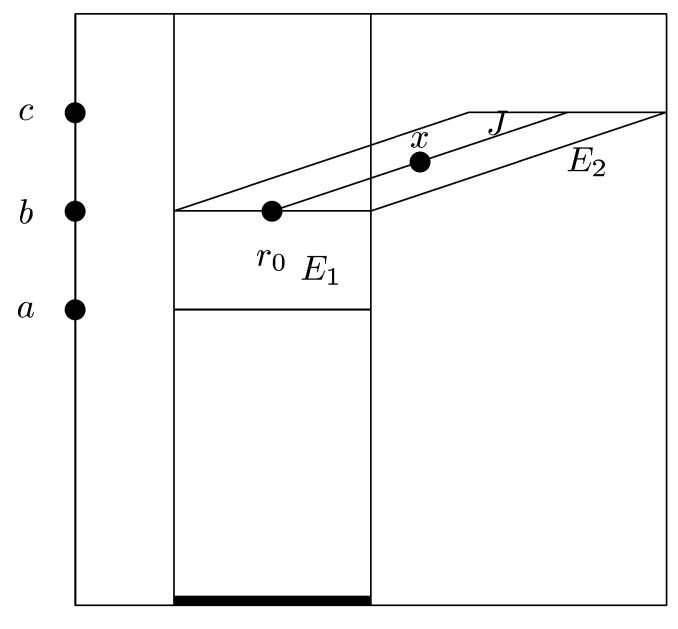

$Q$

Рис. 2

ДоказАТЕльство. Условие (6) означает, что $H_{p}^{\omega}\left(I^{d}\right)=B_{p, 1}^{s}\left(I^{d}\right)$ для $s=(d-$ $1) / p$. Поскольку непрерьвные функции плотны в $B_{p, 1}^{s}\left(I^{d}\right)$, то достаточность следует стандартным образом из оценки слабого типа для максимального оператора.

Покажем теперь необходимость. Пусть (6) не выполнено. Тогда существует неотрицательная функция $f \in \widetilde{H}_{p}^{\omega}\left(I^{d-1}\right), f \notin L^{\infty}\left(I^{d-1}\right)$. Определим функцию $F \in \widetilde{H}_{p}^{\omega}\left(I^{d-1}\right)$ так же, как и ранее в $(3)$, и положим

$$
f_{0}\left(x_{1}, \ldots, x_{d}\right) \equiv F\left(x_{1}, \ldots, x_{d-1}\right) .
$$

Если $x=\left(x_{1}, \ldots, x_{d}\right) \in I^{d}$, то в любой окрестности точки $\widehat{x}_{d}=\left(x_{1}, \ldots, x_{d-1}\right)$ найдется шар $Q$ такой, что средние $|Q|^{-1} \int_{Q} F$ достаточно велики. Тогда легко сконструировать гибкий цилиндр $E \ni x, E \subset I^{d}$, для которого

$$
|E|^{-1} \int_{E \ni x} f_{0} \geqslant(2|Q|)^{-1} \int_{Q} F
$$

Действительно, пусть $r=\left(x_{1}, \ldots, x_{d-1}, 0\right)$ - центр $Q$, а $a, b$ и $c$ такие, что $b-a=c-b$ и $x_{d} \in(b, c)$, причем $(a, c) \in(0,1)$. Проведем через точку $r_{0}=$ $\left(x_{1}, \ldots, x_{d-1}, b\right)$ и $x$ отрезок $J$, с началом в точке $r_{0}$, так, чтобы его проекция на ось $X_{d}$ совпала с отрезком $[b, c]$. Обозначим теперь через $E_{1}$ объединение шаров с центрами в точках $J$, конгруентных $Q$, и положим $E_{2} \equiv(a, b) \times Q,\left|E_{1}\right|=\left|E_{2}\right|$. Через $E$ обозначим внутренность множества $E_{1} \cup E_{2}$ (см. рис. 2 ). Очевидно, $E \ni x$, $|E|=2\left|E_{2}\right|$.

Тогда

$$
|E|^{-1} \int_{E \ni x} f_{0} \geqslant\left(2\left|E_{2}\right|\right)^{-1} \int_{E_{2}} f_{0}=(2|Q|)^{-1} \int_{Q} F
$$


следовательно, интегральные средние $|E|^{-1} \int_{E \ni x} f_{0}$ достаточно велики, что приводит к недифференцируемости интеграла. Теорема 1 доказана.

Случай $p=1$. Введем дополнительные обозначения. Для заданной на отрезке $[a ; b]$ функции $\phi$ через var $\phi$ обозначим вариацию функции $\phi$, а через

$$
V([a ; b], \phi)=\inf _{\psi \sim \phi} \operatorname{var} \psi
$$

обозначим существенную вариацию $\phi$ на $[a ; b]$. Через

$$
V([a ; b], f(\cdot, t)) \text { и } V([a ; b], f(t, \cdot))
$$

обозначим сушественные вариации функции $f$ по первой и второй координатам соответственно, при фиксированном значении другой переменной, равном $t$. Наконец, через $W([a ; b], \phi)$ обозначим существенное колебание функции $\phi$ на $[a ; b]$.

В $\S 5$ мы покажем, что в случае, когда размерность пространства больше двойки, не сушествует нетривиального ограничения на гладкость, которое гарантирует дифференцирование интегралов базисами из выпуклых множеств. Аналогичным образом это утверждение доказывается и для базиса из гибких цилиндров.

Двумерный случай более содержателен. Мы не только установим дифференцируемость, но и получим некоторую удовлетворительную оценку п.в. скорости дифференцирования.

Tеорема 2. Пусть $f \in \operatorname{Lip}(1,1)\left(I^{2}\right)$. Тогда

$$
\left|\left\{x \in I^{2}: \mathscr{M}_{\mathscr{B}} f(x)>\lambda\right\}\right| \ll \lambda^{-1}\left[\sup _{h>0} \frac{\omega(f ; h)_{1}}{h}+\|f\|_{1}\right], \quad \lambda>0,
$$

интеграл от $f$ дифферениируется базисом $\mathscr{B}$ и для почти всех $x \in I^{2}$ справедлива оченка

$$
|P|^{-1} \int_{\mathscr{B} \ni P \ni x}|f(y)-f(x)| d y=O_{x}\{\operatorname{diam}(P) \psi(\operatorname{diam}(P))\},
$$

где $\psi(t)$ - невозрастающая положительная функиия, для которой

$$
\int_{0}^{1} \frac{d t}{t \psi(t)}<\infty
$$

Доказательству теоремы 2 предпошлем ряд предварительных утверждений. Начнем с хорошо известной теоремы Харди-Литлвуда.

Теорема А (см. [18, п. 4.8.2]). Если для $f \in L\left(I^{1}\right)$ выполнено условие

$$
\omega(f ; h)_{1} \leqslant \alpha h, \quad 0<h<1
$$

то $f$ эквивалентна некоторой функиии, которую мы снова обозначим через $f$, ограниченной вариации на $I^{1}$ и

$$
\operatorname{var} f \leqslant \alpha
$$


Лемма 2. Пусть $f \in \operatorname{Lip}(1,1)\left(I^{2}\right)$. Тогда найдется неотрицательная функиия $v(t)$ такая, что

$$
V([0,1], f(\cdot, t))+V([0,1], f(t, \cdot)) \ll v(t), \quad t \in[0,1]
$$

$u$

$$
\int_{0}^{1} v(t) d t \ll \sup _{h>0} \frac{\omega(f ; h)_{1}}{h} .
$$

ДОКАЗАТЕЛЬСТво. Поскольку $\omega_{j}(f ; 2 h)_{1} \leqslant 2 \omega_{j}(f ; h)_{1}$, то последовательность $2^{n} \omega_{2}\left(f ; 2^{-n}\right)_{1}$ является неубьваюшей.

Тогда с учетом (5) получим

$$
\begin{aligned}
2^{n} \omega_{2}\left(f ; 2^{-n}\right)_{1} & \gg \int_{0}^{1} 2^{2 n} \int_{0}^{2^{-n}} \int_{0}^{1}\left|f\left(x_{1}, x_{2}+h\right)-f\left(x_{1}, x_{2}\right)\right| d x_{2} d h d x_{1} \\
& \gg \int_{0}^{1} 2^{n} \omega_{2},\left(f\left(x_{1}, \cdot\right) ; 2^{-n}\right)_{1} d x_{1} .
\end{aligned}
$$

Таким образом,

$$
\sup _{n \geqslant 1} \int_{0}^{1} 2^{n} \omega_{2}\left(f\left(x_{1}, \cdot\right) ; 2^{-n}\right)_{1} d x_{1} \ll \sup _{h>0} \frac{\omega(f ; h)_{1}}{h}<\infty .
$$

Отсюда по теореме Леви получим существование функции

$$
v_{2}(t)=\lim _{n \rightarrow \infty} 2^{n} \omega_{2}\left(f(t, \cdot) ; 2^{-n}\right)_{1}
$$

такой, что

$$
\int_{0}^{1} v_{2}(t) d t \ll \sup _{h>0} \frac{\omega(f ; h)_{1}}{h}
$$

и для п.в. $t \in[0,1]$

$$
\sup _{h>0} \frac{\omega(f(t, \cdot) ; h)_{1}}{h} \ll v_{2}(t) .
$$

По теореме А, функция $f(t, \cdot)$ при п.в. $t$ является функцией ограниченной существенной вариации, с оценкой вариации

$$
V(f(t, \cdot) ;[0,1]) \ll v_{2}(t), \quad t \in[0,1] .
$$

Аналогичньм образом строится функция $v_{1}(t)$, и полагаем $v=v_{1}+v_{2}$. Лемма 2 доказана. 


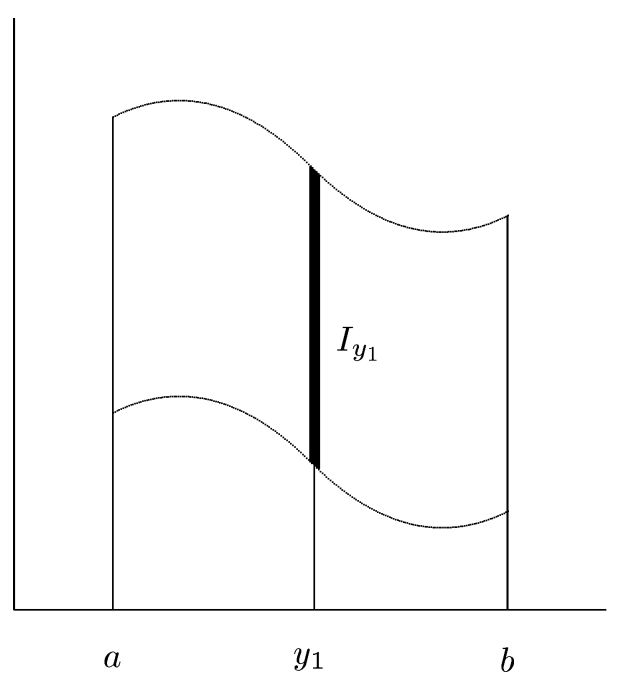

Рис. 3

Перейдем непосредственно к доказательству теоремы 2. Пусть $P \in \mathscr{B}_{2}, x \in P$. Обозначим через $[a, b]$ проекцию $P$ на ось $X$ и введем в рассмотрение интервал $I_{y_{1}}=\left\{y_{2}:\left(y_{1}, y_{2}\right) \in P\right\}$, где $y_{1} \in[a ; b]$ (см. рис. 3$)$.

Поскольку $|P|=(b-a)\left|I_{y_{1}}\right|$, то

$$
\begin{aligned}
& |P|^{-1} \int_{P \ni x}|f(y)| d y \\
& \quad \ll \frac{1}{b-a} \int_{a}^{b} \frac{1}{\left|I_{y_{1}}\right|} \int_{I_{y_{1}}}\left|f\left(y_{1}, x_{2}\right)-f\left(y_{1}, y_{2}\right)\right| d y_{2} d y_{1}+\frac{1}{b-a} \int_{a}^{b}\left|f\left(y_{1}, x_{2}\right)\right| d y_{1} .
\end{aligned}
$$

Так как $\left|f\left(y_{1}, x_{2}\right)-f\left(y_{1}, y_{2}\right)\right| \leqslant V\left(f\left(y_{1}, \cdot\right) ;[0,1]\right)$, то

$$
\mathscr{M}_{\mathscr{B}} f \ll \mathscr{M}^{2} v+\mathscr{M}^{1} f .
$$

Теперь требуемое соотношение (7) следует из (9), (10) и (11).

Осталось получить (8). Продолжим $f$ равной нулю вне $I^{2}$, и положим для $x=$ $\left(x_{1} ; x_{2}\right)$ и $x_{2} \in\left[(k-1) 2^{-n} ; k 2^{-n}\right), 1 \leqslant k \leqslant 2^{n}$,

$$
W_{n} f(x)=W\left(\left[(k-1) 2^{-n} ; k 2^{-n}\right], f\left(x_{1} ; \cdot\right)\right) .
$$

Очевидно, $W_{1} f(x) \leqslant V\left([0,1], f\left(x_{1}, \cdot\right)\right)$ и $W_{n} f(x)$ представляет собой невозрастающую последовательность суммируемых функций.

Оценим $\left\|W_{n} f\right\|_{1}$. Имеем для п.в. $x_{1} \in[0,1]$

$$
\begin{aligned}
\sum_{k=1}^{2^{n}} W\left(\left[(k-1) 2^{-n} ; k 2^{-n}\right], f\left(x_{1} ; \cdot\right)\right) & \leqslant \sum_{k=1}^{2^{n}} V\left(\left[(k-1) 2^{-n} ; k 2^{-n}\right], f\left(x_{1} ; \cdot\right)\right) \\
& \leqslant V\left([0,1], f\left(x_{1}, \cdot\right)\right) \ll v\left(x_{1}\right),
\end{aligned}
$$


и, таким образом,

$$
\begin{aligned}
\left\|W_{n} f\right\|_{1} & =\int_{0}^{1} \sum_{k=1}^{2^{n}} W\left(\left[(k-1) 2^{-n} ; k 2^{-n}\right], f\left(x_{1} ; \cdot\right)\right) d x_{1} 2^{-n} \\
& \ll 2^{-n}\|v\|_{1} \ll 2^{-n} \sup _{h>0} \frac{\omega(f ; h)_{1}}{h} .
\end{aligned}
$$

Положим теперь

$$
S f(x)=\sum_{n=0}^{\infty} \frac{W_{n} f\left(x_{1}, x_{2}-2^{-n}\right)+W_{n} f\left(x_{1}, x_{2}\right)+W_{n} f\left(x_{1}, x_{2}+2^{-n}\right)}{2^{-n} \psi\left(2^{-n}\right)} .
$$

Тогда

$$
\|S f\|_{1} \ll \sum_{n=1}^{\infty} \frac{\left\|W_{n} f\right\|_{1}}{2^{-n} \psi\left(2^{-n}\right)} \ll \sum_{n=1}^{\infty} \frac{1}{\psi\left(2^{-n}\right)} \ll \int_{0}^{1} \frac{d t}{t \psi(t)}<\infty .
$$

Пусть теперь $x \in P, \operatorname{diam}(P) \leqslant 2^{-3}$. Через $\operatorname{Pr}$ мы обозначим проекцию $P$ на ось $Y$. Тогда для некоторого $n, 2^{-n} \leqslant|\operatorname{Pr}|<2^{-n+1}$, и для некоторого $k$ справедливо неравенство $(k-1) 2^{-n} \leqslant x_{2}<k 2^{-n}$.

Теперь возможны два случая:

$$
\operatorname{Pr} \in\left[(k-2) 2^{-n} ; k 2^{-n}\right] \text { или } \operatorname{Pr} \in\left[(k-1) 2^{-n} ;(k+1) 2^{-n}\right] .
$$

Пусть $y_{1} \in[0,1]$ и $y_{2} \in \operatorname{Pr}$. В первом случае получим

$$
\begin{aligned}
& \left|f\left(y_{1}, x_{2}\right)-f\left(y_{1}, y_{2}\right)\right| \\
& \quad \leqslant W\left(\left[(k-1) 2^{-n} ; k 2^{-n}\right], f\left(y_{1} ; \cdot\right)\right)+W\left(\left[(k-2) 2^{-n} ;(k-1) 2^{-n}\right], f\left(y_{1} ; \cdot\right)\right) \\
& \quad=W_{n} f\left(y_{1}, x_{2}\right)+W_{n} f\left(y_{1}, x_{2}-2^{-n}\right) .
\end{aligned}
$$

Во втором

$$
\begin{aligned}
& \left|f\left(y_{1}, x_{2}\right)-f\left(y_{1}, y_{2}\right)\right| \\
& \quad \leqslant W\left(\left[(k-1) 2^{-n} ; k 2^{-n}\right], f\left(y_{1} ; \cdot\right)\right)+W\left(\left[k 2^{-n} ;(k+1) 2^{-n}\right], f\left(y_{1} ; \cdot\right)\right) \\
& \quad=W_{n} f\left(y_{1}, x_{2}\right)+W_{n} f\left(y_{1}, x_{2}+2^{-n}\right) .
\end{aligned}
$$

В результате, почти всюду

$$
\begin{aligned}
& \left|f\left(y_{1}, x_{2}\right)-f\left(y_{1}, y_{2}\right)\right| \\
& \quad \ll W_{n} f\left(y_{1}, x_{2}\right)+W_{n} f\left(y_{1}, x_{2}-2^{-n}\right)+W_{n} f\left(y_{1}, x_{2}+2^{-n}\right) \\
& \quad \ll 2^{-n} \psi\left(2^{-n}\right) S f\left(y_{1}, x_{2}\right) .
\end{aligned}
$$

Далее,

$$
\begin{aligned}
|P|^{-1} \int_{P}|f(x)-f(y)| d y \ll & \frac{1}{b-a} \int_{a}^{b} \frac{1}{\left|I_{y_{1}}\right|} \int_{I_{y_{1}}}\left|f\left(y_{1}, x_{2}\right)-f\left(y_{1}, y_{2}\right)\right| d y_{2} d y_{1} \\
& +\frac{1}{b-a} \int_{a}^{b}\left|f\left(y_{1}, x_{2}\right)-f\left(x_{1}, x_{2}\right)\right| d y_{1} \\
\equiv & \Sigma_{1}+\Sigma_{2} .
\end{aligned}
$$


Теперь

$$
\Sigma_{1} \leqslant \frac{1}{b-a} \int_{a}^{b} S f\left(y_{1}, x_{2}\right) d y_{1} 2^{n} \psi\left(2^{-n}\right) \leqslant \mathscr{M}^{2}(S f)(x) 2^{n} \psi\left(2^{-n}\right) .
$$

Обозначим

$$
\mathscr{M}^{\star} f(x)=\sup _{I \ni x} \frac{V\left(I ; f\left(\cdot ; x_{2}\right)\right)}{|I|} .
$$

Функция $\mathscr{M}^{\star} f$ обладает слабым типом (1-1) как максимальная функция ХардиЛитлвуда от меры (см., например, [19, п. 4.1])

$$
\left|\left\{x \in I^{2}: \mathscr{M}^{\star} f(x)>\lambda\right\}\right| \ll \frac{1}{\lambda} \int_{0}^{1} V\left([0,1] ; f\left(\cdot ; x_{2}\right)\right) d x_{2}, \quad \lambda>0 .
$$

Тогда с учетом леммы 2 получим $\Sigma_{2} \ll \mathscr{M}^{\star} f(x)(b-a)$, где $\mathscr{M}^{\star} f(x)<\infty$ п.в. В итоге,

$$
|P|^{-1} \int_{P \ni x}|f(x)-f(y)| d y \ll \mathscr{M}^{2}(S f)(x) \operatorname{diam}(P) \psi(\operatorname{diam}(P))+\mathscr{M}^{\star} f(x) \operatorname{diam}(P) .
$$

Так как, $\mathscr{M}^{2}(S f)(x)$ и $\mathscr{M}^{\star} f(x)$ конечны почти повсюду, то теорема 2 доказана.

Итак, в двумерном случае наилучшая гладкость гарантирует дифференцируемость интеграла базисом из гибких цилиндров. Оказывается, никакой другой гладкости, кроме наилучшей, уже недостаточно. Мы докажем это утверждение в следуюшем параграфе для базиса из выпуклых множеств. Для базиса из гибких цилиндров оно доказьвается аналогично.

\section{§5. Базис из выпуклых множеств}

Оценки сверху. В предыдушем параграфе мы установили положительные результаты для базиса из гибких цилиндров. Эти результаты переносятся на базис из выпуклых множеств, поскольку вокруг любого выпуклого множества можно описать гибкий цилиндр сравнимой с ним меры и диаметром. Приведем полное доказательство этого факта в двумерном случае, и опишем схему доказательства в обшем случае.

Лемма 3. Для любого ограниченного выпуклого множества $Q$ на плоскости существует параллелограмм $P$, с парой сторон параллельных оси $Y$, такой, что

$$
Q \subset P, \quad|P| \leqslant 2|Q|, \quad \operatorname{diam}(P) \leqslant 3 \operatorname{diam}(Q) .
$$




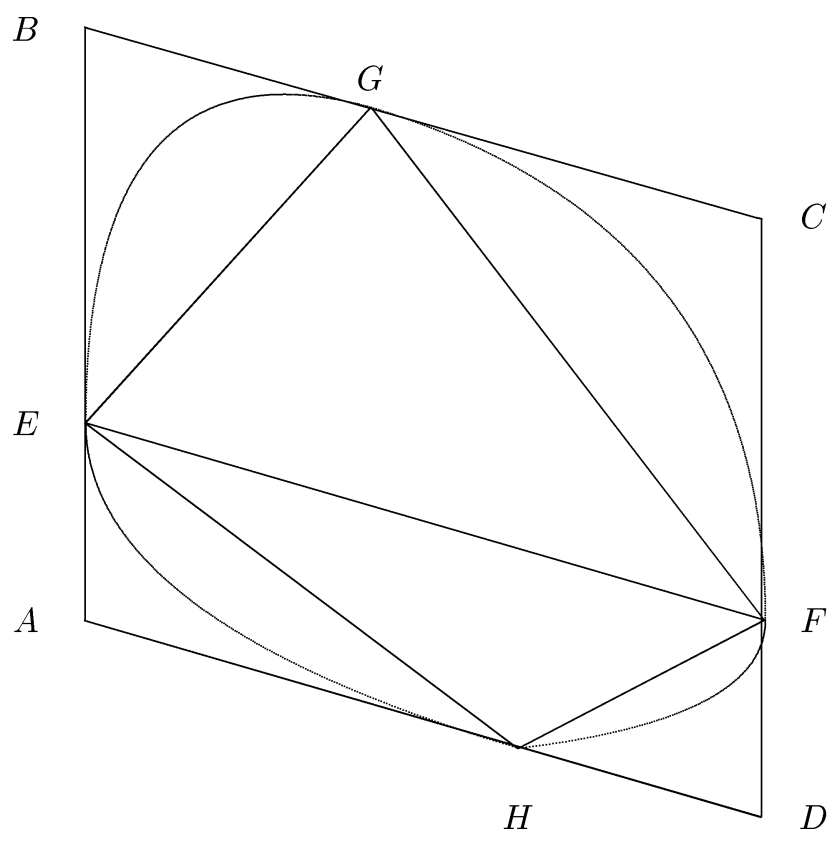

Рис. 4

ДокАЗАТЕЛЬСтво. Пусть (см. рис. 4) $A B$ и $C D$ - опорные прямые параллельные оси $Y$ и $E \in B A \cap \partial Q ; F \in C D \cap \partial Q$.

Проведем опорные прямые $B C$ и $A D$ параллельно $E F$, и пусть

$$
G \in B C \cap \partial Q ; \quad H \in A D \cap \partial Q .
$$

Из определения выпуклости $Q$ получим, что $E G F H \subset Q$. Очевидно,

$$
2|E G F|=|E B C F| ; \quad 2|E H F|=|A E F D| .
$$

Положим теперь $P=A B C D$. Тогда $2|E G F H|=|P|$. Поскольку $E G F H \subset$ $Q \subset P$, то $|P| \leqslant 2|Q|$.

Далее, не ограничивая общности, считаем $|C F| \geqslant 2^{-1}|C D|$ и $\angle B C F \geqslant \pi / 2$. Тогда $|G F| \geqslant 2^{-1}|C D|$ и, таким образом, $|C D| \leqslant 2|G F| \leqslant 2 \operatorname{diam}(Q)$. Кроме того, $|B C|=|E F| \leqslant \operatorname{diam}(Q)$. В итоге,

$$
\operatorname{diam}(Q) \leqslant|B C|+|C D| \leqslant 3 \operatorname{diam}(Q),
$$

что и доказывает лемму 3.

В обшем случае, можно привлечь стандартные рассуждения с использованием аффинных преобразований. Поскольку вокруг любого выпуклого тела можно описать сравнимый с ним эллипсоид (см., например, [20, с. 40]), то достаточно ими и ограничиться, при этом предполагаем центр эллипсоида расположенным в начале координат. 
Выбрав $\nu$-ю координатную ось, проведем к эллипсоиду опорные гиперплоскости $\Pi_{1}$ и $\Pi_{2}$, перпендикулярные этой оси. Точки касания соединим отрезком $J$. Афффинным преобразованием $\mathscr{H}$ переведем эллипсоид в шар $B$. При этом отрезок $J$ перейдет в отрезок $\mathscr{H}(J)$, а гиперплоскости $\Pi_{1}$ и $\Pi_{2}$ в гиперплоскости $\mathscr{H}\left(\Pi_{1}\right)$ и $\mathscr{H}\left(\Pi_{2}\right)$. Пусть $P_{0}$ обозначает цилиндр с осью $\mathscr{H}(J)$, описанный вокруг $B$ (его основаниями являются шары, расположенные на $\mathscr{H}\left(\Pi_{1}\right)$ и $\left.\mathscr{H}\left(\Pi_{2}\right)\right)$. Обратньм преобразованием $\mathscr{H}^{-1}$ переведем шар снова в эллипсоид. Из обших свойств аф̆финных преобразований следует, что цилиндр $P_{0}$ отобразился в гибкий цилиндр $P \equiv \mathscr{H}^{-1}\left(P_{0}\right)$ сравнимой с ним меры. Сравнимость диаметров доказывается так же, как и в лемме 3, с учетом многомерной специфики.

Таким образом, нами установлены положительные результаты для базиса из выпуклых множеств. В случае $p=1$, как мы уже отмечали, эти результаты можно дополнить контрпримерами, устанавливающими их точность. Однако, нам не удалось установить окончательность этих результатов в случае $1<p<\infty$. Вполне возможно, что в этом случае положительные результаты могут быть улучшены. Предполагаемое доказательство этого факта нам представляется достаточно нетривиальным, так как оно должно бы существенно опираться на свойства, порождаемые выпуклостью множеств из базиса.

Оценки снизу. Случай $p=1$. Итак, покажем, что в случае, когда размерность пространства больше двойки, не сушествует нетривиального ограничения на гладкость, которое гарантирует дифференцирование интегралов базисами из выпуклых множеств.

Теорема 3. Для любого $d \geqslant 3$ существует функиия $f \in L\left(I^{d}\right)$ с условием

$$
\omega(f, h)_{1}=O(h),
$$

такая, что

$$
\limsup _{\operatorname{diam}(Q) \rightarrow 0}|Q|^{-1} \int_{Q \ni x} f(y) d y=+\infty
$$

n.в. на $I^{d}$.

ДокАЗАтЕльство. Не ограничивая общности, считаем $d=3$. Разобьем $I^{3}$ на $m^{3}$ попарно непересекающихся одинаковых кубов $I_{k}^{m},\left|I_{k}^{m}\right|=m^{-3}$. В каждый куб $I_{k}^{m}$ поместим концентрический с ним куб $Q_{k}^{m},\left|Q_{k}^{m}\right|=2^{-3 m}$, и определим функции $f_{k}^{m}=2^{m} \chi_{Q_{k}^{m}}$ И

$$
f=\sum_{m=1}^{\infty} \sum_{k=1}^{m^{3}} f_{k}^{m} .
$$

Очевидно,

$$
\|f\|_{1} \leqslant \sum_{m=1}^{\infty} 2^{m} m^{3} 2^{-3 m}<\infty .
$$

Покажем, что для любого $x \in I_{k}^{m}$ можно подобрать выпуклое множество $Q(x) \ni$ $x$ такое, что $\operatorname{diam}(Q(x)) \ll 1 / m$ и

$$
|Q(x)|^{-1} \int_{Q(x)} f_{k}^{m}(y) d y \gg m,
$$


что влечет (13).

Чтобы построить $Q(x)$, поступим следующим образом. Пусть $x \in I_{k}^{m}$. Соединим точку $x$ с центром $I_{k}^{m}$ отрезком $J$. Опишем около $Q_{k}^{m}$ шар $B$, и через $Q(x)$ обозначим объединение всех шаров с центрами на $J$, полученных сдвигом $B$ (см. рис. 5).

Очевидно, $Q(x) \supset Q_{k}^{m}, \operatorname{diam}(Q(x)) \ll \operatorname{diam}\left(I_{k}^{m}\right)$. С другой стороны, $|Q(x)| \ll$ $(\operatorname{diam}(B))^{2}|J| \ll\left|Q_{k}^{m}\right|^{2 / 3}|J| \ll\left|Q_{k}^{m}\right|^{2 / 3} m^{-1}$. Тогда

$$
|Q(x)|^{-1} \int_{Q(x)} f_{k}^{m}(y) d y \gg \frac{2^{m}\left|Q_{k}^{m}\right|}{\left|Q_{k}^{m}\right|^{2 / 3} m^{-1}}=m
$$

что и требовалось получить.

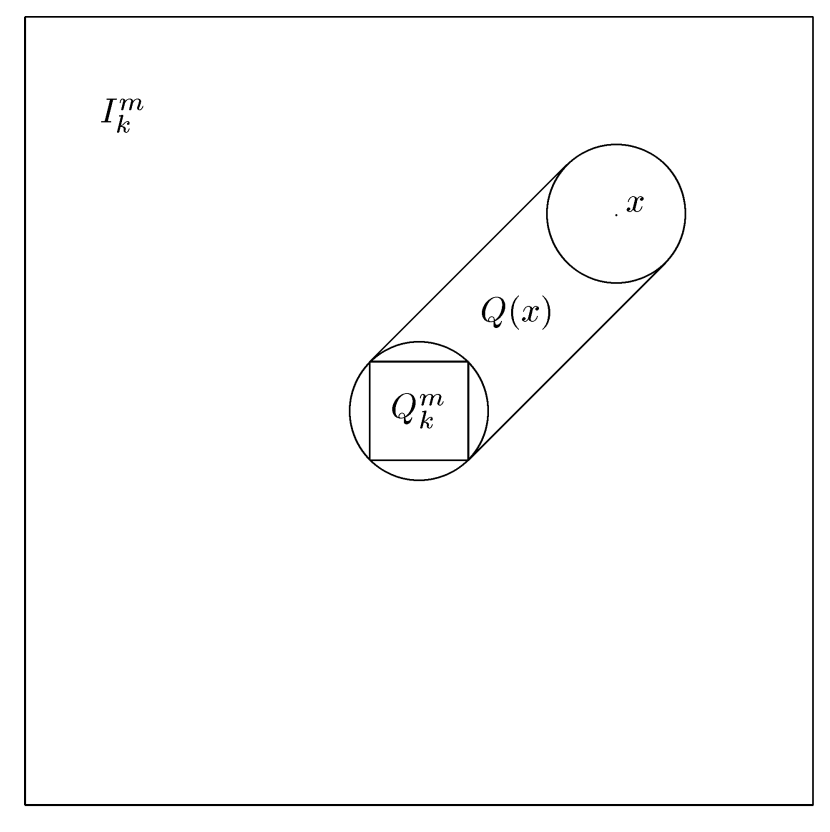

Рис. 5

Оценим теперь модуль непрерывности функции $f$. Для $2^{-m-1} \leqslant h<2^{-m}$ имеем

$$
\begin{aligned}
\omega_{j}(f ; h)_{1} & \leqslant \sum_{n=1}^{m} \sum_{k=1}^{n^{3}} \omega_{j}\left(f_{k}^{n} ; h\right)+2 \sum_{n=m+1}^{\infty} \sum_{k=1}^{n^{3}}\left\|f_{k}^{n}\right\|_{1} \\
& \ll h \sum_{n=1}^{m} n^{3} 2^{n} 2^{-2 n}+\sum_{n=m+1}^{\infty} n^{3} 2^{n} 2^{-3 n} \\
& \ll h \sum_{n=1}^{m} n^{3} 2^{-n}+2^{-m} \sum_{n=m+1}^{\infty} n^{3} 2^{-n},
\end{aligned}
$$


что влечет (12). Теорема 3 доказана.

Далее, в двумерном случае наилучшая гладкость гарантирует дифференцируемость интеграла базисом из гибких цилиндров. Покажем, что никакой другой гладкости, кроме наилучшей, уже недостаточно.

Теорема 4. Пусть $\omega(h)$ - модуль непрерывности такой, что $\omega(h) / h \rightarrow \infty$, $h \rightarrow+0$. Тогда существует функиия $f \in L\left(I^{2}\right)$ такая, что

$$
\omega(f, h)_{1}=O(\omega(h))
$$

$u$

$$
\limsup _{\operatorname{diam}(Q) \rightarrow 0}|Q|^{-1} \int_{Q \ni x} f(y) d y=+\infty
$$

почти всюду на $I^{2}$.

ДоКАЗАТЕЛьство. Выберем последовательность $a_{k}$ так, чтобы она монотонно убцвала к нулю и

$$
a_{0}=1, \quad a_{n} \leqslant 1 / n, \quad \omega\left(a_{n}\right) \geqslant a_{n} n^{3}, \quad 2 a_{n+1} \leqslant a_{n} .
$$

Разобьем $I^{2}$ на $n^{2}$ неперекрывающихся одинаковых квадратов $I_{k}^{n},\left|I_{k}^{n}\right|=n^{-2}$. В каждый из квадратов $I_{k}^{n}$ поместим концентрический с ним квадрат $Q_{k}^{n},\left|Q_{k}^{n}\right|=a_{n}^{2}$. Положим $f_{k}^{n}=a_{n}{ }^{-1} \chi_{Q_{k}^{n}}$ и

$$
f=\sum_{n=1}^{\infty} \sum_{k=1}^{n^{2}} f_{k}^{n}
$$

Тогда, учитывая (15), получим

$$
\|f\|_{1} \leqslant \sum_{n=1}^{\infty} n^{2} a_{n}<\infty .
$$

Как и в теореме 3 (см. рис. 5 ), нетрудно убедиться, что для каждого $x \in I_{k}^{n}$ можно подобрать прямоугольник $R, x \in R, \operatorname{diam}(R) \ll 1 / n$ и $|R| \ll a_{n} / n$ такой, что

$$
|R|^{-1} \int_{R} f_{k}^{n}(y) d y \gg \frac{a_{n}^{-1} a_{n}^{2}}{a_{n} / n}=n,
$$

откуда получим (14). Оценим гладкость функции $f$. Пусть $a_{m+1}<h \leqslant a_{m}$. Тогда

$$
\begin{aligned}
\omega_{j}(f ; h)_{1} & \leqslant \sum_{n=1}^{m} \sum_{k=1}^{n^{2}} \omega_{j}\left(f_{k}^{n} ; h\right)_{1}+2 \sum_{n=m+1}^{\infty} \sum_{k=1}^{n^{2}}\left\|f_{k}^{n}\right\|_{1} \\
& \ll h \sum_{n=1}^{m} a_{n} \frac{1}{a_{n}} n^{2}+\sum_{n=m+1}^{\infty} a_{n}^{2} \frac{1}{a_{n}} n^{2} \ll h m^{3}+\sum_{n=m+1}^{\infty} a_{n} n^{2} .
\end{aligned}
$$


Из (15) получим

$$
\sum_{n=m+1}^{\infty} a_{n} n^{2} \ll a_{m+1}(m+1)^{3} \ll h m^{3},
$$

следовательно, $\omega_{j}(f ; h)_{1} \ll m^{3} h$. Опять же, учитывая (12), будем иметь

$$
\frac{\omega(h)}{h} \geqslant \frac{\omega\left(a_{m}\right)}{a_{m}} \geqslant m^{3},
$$

откуда $\omega_{j}(f ; h)_{1} \ll \omega(h)$. Теорема 4 доказана.

Случай $1<p<2$.

ТЕОРемА 5. Пусть $0 \leqslant \sigma(t) \uparrow \infty$ так медленно, чтобъ

$$
\lim _{n \rightarrow \infty} \frac{\sigma\left(2^{n+1}\right)}{\sigma\left(2^{n}\right)}=1 .
$$

Тогда существует функиия $f \in L^{p}\left(I^{2}\right), 1<p<2$, такая, что

$$
\omega(f ; h)_{p}=O\left\{h^{2 / p-1} \sigma\left(\frac{1}{h}\right)\right\}, \quad h \in(0,1),
$$

и интеграл от $f$ не дифференцируется базисом из произвольно ориентированных прямоугольников.

ДокАЗАТЕЛЬСтво. Из (16) следует, что для некоторого $A>0$ и натурального $n_{0}$

$$
\sqrt{\sigma\left(2^{n}\right)} \leqslant A\left(\frac{3}{2}\right)^{n}, \quad n \geqslant n_{0}
$$

Не ограничивая общности, будем предполагать $\sigma(1)=A_{0}=n_{0}=1$.

Положим $m_{n}=\left[\sqrt{\sigma\left(2^{n}\right)}\right]$, и разобьем $I^{2}$ на $m_{n}^{2}$ одинаковых неперекрывающихся квадратов $I_{k}^{n}, k=1, \ldots, m_{n}$.

Через $Q_{k}^{n}$ обозначим концентрический с $I_{k}^{n}$ куб такой, что

$$
\left|Q_{k}^{n}\right|=2^{-2 n}, \quad k=1, \ldots, m_{n}
$$

В силу (18) $Q_{k}^{n} \subset I_{k}^{n}$.

Положим теперь

$$
f_{k}^{n}=2^{n} \chi_{Q_{k}^{n}} \quad \text { и } f=\sum_{n=1}^{\infty} \sum_{k=1}^{m_{n}} f_{k}^{n}
$$

Точно так же, как и в теореме 3 (см. рис. 5 ), для любого $x \in I_{k}^{n}$ найдется прямоугольник $R \ni x, R \ni Q_{k}^{n}$ такой, что

$$
|R|^{-1} \int_{R} f_{k}^{n}(y) d y \gg \frac{2^{-n}\left|Q_{k}^{n}\right|}{2^{-n} \cdot 1 / m_{n}}=m_{n} \rightarrow \infty, \quad n \rightarrow \infty .
$$


Следовательно, интеграл от $f$ не дифференцируем базисом из прямоугольников.

Оценим теперь модуль непрерьвности функции $f$. Для $2^{-s-1}<h \leqslant 2^{-s}$ имеем

$$
\begin{aligned}
\omega_{j}(f ; h)_{p} & \ll \sum_{n=1}^{s} \sum_{k=1}^{m_{n}^{2}} \omega_{j}\left(f_{k}^{n} ; h\right)_{p}+\sum_{n=s+1}^{\infty} \sum_{k=1}^{m_{n}^{2}}\left\|f_{k}^{n}\right\|_{p} \\
& \ll h^{1 / p} \sum_{n=1}^{s} 2^{n}\left(2^{-n}\right)^{1 / p} m_{n}^{2}+\sum_{n=s+1}^{\infty} 2^{n}\left(2^{-2 n}\right)^{1 / p} m_{n}^{2} \\
& \ll h^{1 / p} \sum_{n=1}^{s}\left(2^{-n}\right)^{1 / p-1} \sigma\left(2^{n}\right)+\sum_{n=s+1}^{\infty}\left(2^{-n}\right)^{2 / p-1} \sigma\left(2^{n}\right) .
\end{aligned}
$$

Так как $1<p<2$, а $\sigma\left(2^{n}\right)$ удовлетворяет (16), то

$$
\begin{aligned}
& \sum_{n=1}^{s}\left(2^{-n}\right)^{1 / p-1} \sigma\left(2^{n}\right) \ll\left(2^{-s}\right)^{1 / p-1} \sigma\left(2^{s}\right) \ll h^{1 / p-1} \sigma\left(\frac{1}{h}\right), \\
& \sum_{n=s}^{\infty}\left(2^{-n}\right)^{2 / p-1} \sigma\left(2^{n}\right) \ll\left(2^{-s}\right)^{2 / p-1} \sigma\left(2^{s}\right) \ll h^{2 / p-1} \sigma\left(\frac{1}{h}\right) .
\end{aligned}
$$

Отсюда

$$
\omega_{j}(f ; h)_{p} \ll h^{1 / p} h^{1 / p-1} \sigma\left(\frac{1}{h}\right)+h^{2 / p-1} \sigma\left(\frac{1}{h}\right) \ll h^{2 / p-1} \sigma\left(\frac{1}{h}\right),
$$

что и дает (17). Теорема доказана.

В связи с этой теоремой можно высказать предположение, что в случае $1<$ $p<2$ базис из всех прямоугольников дифференцирует класс $\operatorname{Lip}(2 / p-1 ; p)\left(I^{2}\right)$, u этот класс не улучшаем. Исследования в этом направлении нам представляются весьма интересной и перспективной задачей.

Случай $2 \leqslant p<\infty$.

Tеорема 6. Существует функция $f \in L^{p}\left(I^{2}\right), p \geqslant 2$, для которой

$$
\omega(f, h)_{p}=O\left\{\log \frac{1}{h} \log \log \frac{1}{h}\right\}^{-1 / p}
$$

и интеграл от $f$ не дифференцируется базисом из выпукльх множеств.

ДокАЗАТЕльство. Как уже отмечалось ранее, тот факт, что указанный базис не дифференцирует ни один класс $L^{p}\left(I^{2}\right)$, был установлен А. Зигмундом еще в 1927 году. В 1934 году, Г. Буземанн и В. Феллер [3] предложили другое доказательство, основанное на полученном ими критерии плотности базисов. Случай $1 \leqslant p<2$, как легко убедиться, сводится к оценке снизу функции распределения соответствуюшего максимального оператора на характеристической функции единичного шара. Кстати, нами в предыдушем параграфе так же использовались эти функции. 
Случай $2 \leqslant p \leqslant \infty$ опирается на конструкцию А.С. Безиковича (см. [1], [2]). Нам также придется использовать подобную конструкцию. Однако, в нашем случае, ситуация осложняется тем, что вместо оценок максимального оператора нам приходится непосредственно конструировать функцию. Отметим, что при подсчете модуля непрерьвности этой функции необходимо учитьвать локальную структуру конструкции А.С. Безиковича. Известные модификации этой конструкции основываются на итеративных построениях и их локальная структура не изучалась.

Итак, перейдем к описанию используемой конструкции.

Для начала, зафиксируем произвольное число $\alpha>0$ и рассмотрим в плоскости последовательность горизонтальных прямых $\left\{a_{k}\right\}_{k=1}^{\infty}$, расположенных в одной плоскости относительно прямой $a_{1}$, причем $\operatorname{dist}\left(a_{1}, a_{k}\right)=(k-1) \alpha(k \in \mathbb{N})$.

Договоримся под уровнем с номером $s$ понимать полосу, заключенную между прямыми $a_{s}$ и $a_{s+1}$.

Рассмотрим на первом уровне равнобедренный прямоугольный треугольник с катетом, лежашим на $a_{1}$ и равным $\alpha$. Продолжим другой катет и гипотенузу до пересечения с $a_{3}$ и соединим полученные точки пересечения с вершинами треугольника, лежащими на $a_{1}$. Описанное построение будем называть "процедурой роста".

Легко проверить, что после применения "процедуры роста" мы на втором уровне получим два треугольника, а на первом - параллелограмм с длиной основания и высот, равньми $\alpha$. Обозначим его через $\Pi(1,1, \alpha)$. Если к каждому из треугольников, полученных на втором уровне, опять применить "процедуру роста", то на третьем уровне мы получим уже четыре треугольника, а на втором два параллелограмма $\Pi(2,1, \alpha), \Pi(2,2, \alpha)$, при этом длина основания каждого параллелограмма будет равняться $\alpha / 2$, а высота $\alpha$ (рис. 6 ) и т. д.

Вообще, если рассмотреть $k$-ый уровень, $k>n$, то на нем в результате применения "процедуры роста" на предыдущем уровне получится $2^{k-1}$ треугольников. Если к каждому из них опять применить "процедуру роста", то получится на $k$-м уровне $2^{k-1}$ параллелограммов $\{\Pi(k, \nu, \alpha)\}_{\nu=1}^{2^{k-1}}$, каждый из которых имеет основание, равное $\alpha 2^{1-k}$, а высоту $\alpha$ (нумерация параллелограммов на фиксированном уровне идет справо налево).

Для любого $n \in \mathbb{N}$ положим

$$
E(n, \alpha)=\bigcup_{k=1}^{n} \bigcup_{\nu=1}^{2^{k-1}} \Pi(k, \nu, \alpha) .
$$

При этом, из геометрических соображений ясно, что для каждого параллелограмма $\Pi(k, \nu, \alpha)$ треугольник, образованный пересечением трех прямых, две из которых проходят через середину стороны параллелограмма $\Pi(k, \nu, \alpha)$, лежашей на $a_{k+1}$, и, соответственно, через вершины его основания, а третья является прямой $a_{1}$, полностью содержится в $E(n, \alpha)$. Обозначим этот треугольник через $\triangle_{\nu}^{k}$. Например, на рис. $6 \triangle A B C=\triangle_{1}^{2} \subset E(2, \alpha)$.

Таким образом, с помощью "процедуры роста" мы построили последовательность множеств $\{E(n, \alpha)\}_{n=1}^{\infty}$. 


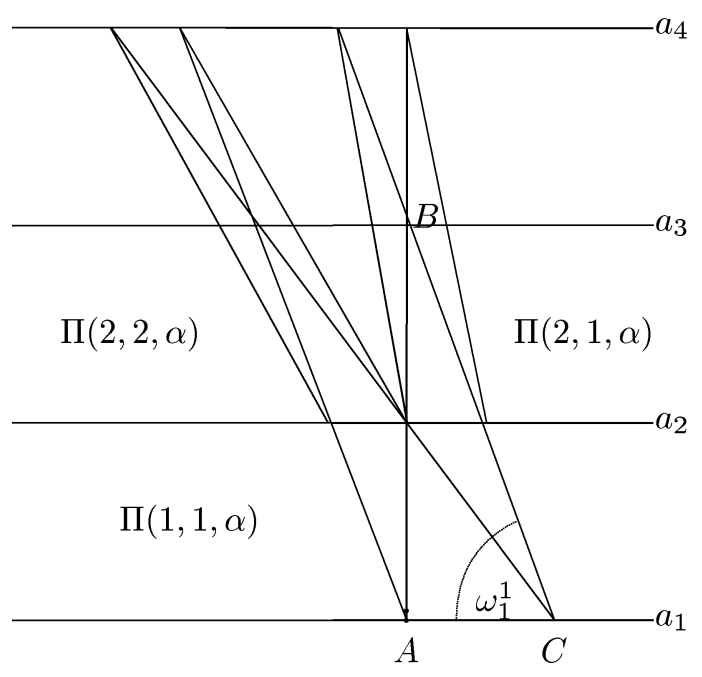

Рис. 6

Из геометрических соображений ясно, что параллелограммы на фиксированном уровне попарно не имеют общих внутренних точек, а потому

$$
|E(n, \alpha)|=\sum_{k=1}^{n} \sum_{\nu=1}^{2^{k-1}}|\Pi(k, \nu, \alpha)|=\sum_{k=1}^{n} \sum_{\nu=1}^{2^{k-1}} \alpha 2^{1-k} \alpha=\alpha^{2} n .
$$

Зафиксируем теперь произвольное множество $E(n, \alpha)$ и параллелограмм $\Pi(n, \nu, \alpha), \nu=1, \ldots, 2^{n-1}$. Проведем через вершины основания треугольника $\triangle_{\nu}^{n}$ прямые, параллельные боковьм сторонам параллелограмма $\Pi(n, \nu, \alpha)$ (рис. 7 ).

Между прямыми $a_{2 n}$ и $a_{4 n}$ у нас получится параллелограмм $R_{\nu}^{n}$. При этом $\left|R_{\nu}^{n}\right|=2^{2-n} n^{2} \alpha^{2}$.

Кроме того, из построений следует, что

$$
R_{\nu}^{n} \cap R_{j}^{n}=\varnothing \quad(\nu \neq j)
$$

Далее, пусть $x \in R_{\nu}^{n}$. Тогда сушествует такой параллелограмм $\widehat{R}_{\nu}^{n}=A B C D$ (см. рис. 7), что

$$
\frac{1}{\left|\widehat{R}_{\nu}^{n}\right|} \int_{\widehat{R}_{\nu}^{n}} \chi_{E(n, \alpha)} \geqslant \frac{\left|\triangle_{\nu}^{n}\right|}{\left|\widehat{R}_{\nu}^{n}\right|}=\frac{n \alpha|A D|}{2|A D|(4 n-1) \alpha} \geqslant \frac{1}{8} .
$$

Здесь мы учли, что $\triangle_{\nu}^{n} \subset E(n, \alpha)$.

Таким образом, если $G(n, \alpha)=\bigcup_{\nu=1}^{2^{n-1}} \widehat{R}_{\nu}^{n}$, то

$$
|G(n, \alpha)| \geqslant\left|\bigcup_{\nu=1}^{2^{n-1}} R_{\nu}^{n}\right|=\sum_{\nu=1}^{2^{n-1}} 2^{2-n} n^{2} \alpha^{2}=2 n^{2} \alpha^{2}
$$




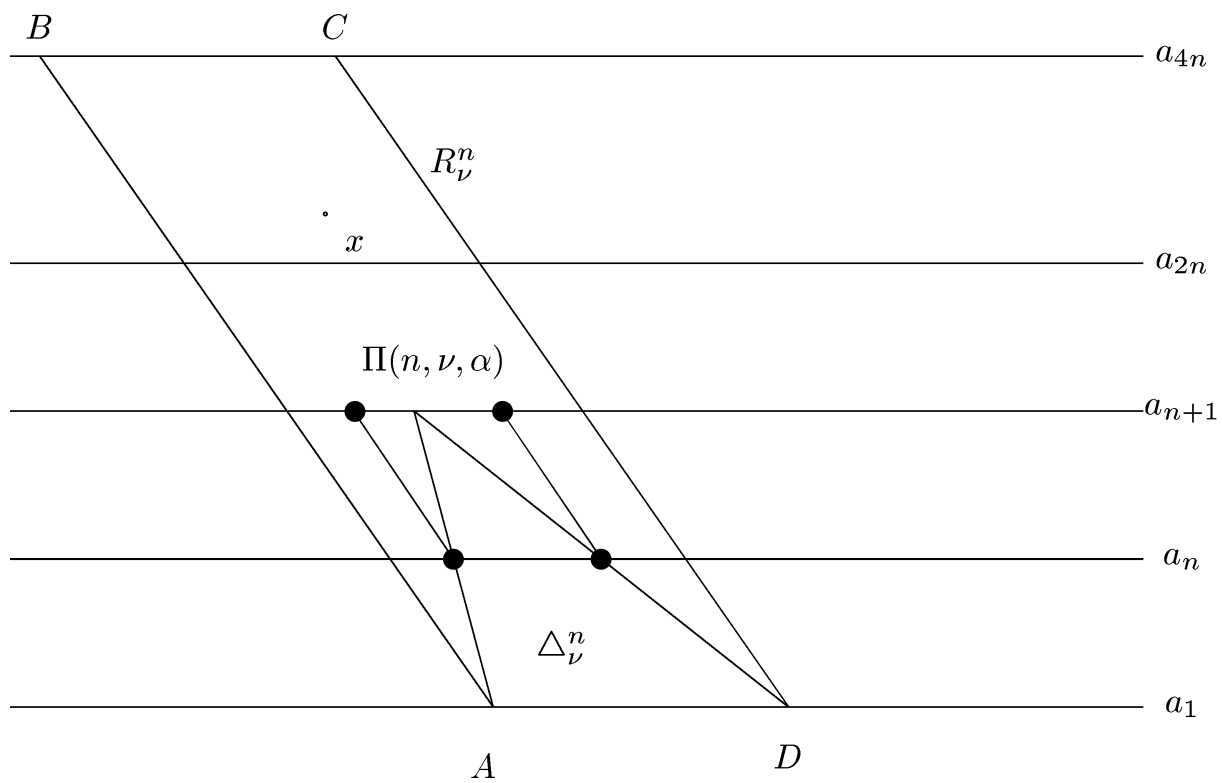

Рис. 7

Теперь конструкция описана полностью. Основное ее свойство заключается в том, что интегральные средние в (19) отделены от нуля, в то время как $\frac{|G(n, \alpha)|}{|E(n, \alpha)|} \rightarrow \infty$,
$n \rightarrow \infty$.

Перейдем к построению функции $f$. По ходу дела нам придется рассматривать достаточно редкие номера конструкций (вместо $n$ брать $2^{n}$ ), и подвергать конструкции уменьшениям за счет выбора различных параметров $\alpha$.

Итак, пусть $\alpha_{n}=\left(2^{n} \sqrt{n}\right)^{-1}$. Тогда

$$
\left|G\left(2^{n}, \alpha_{n}\right)\right| \geqslant 22^{2 n}\left(2^{n} \sqrt{n}\right)^{-2}=\frac{2}{n},
$$

и, таким образом,

$$
\sum_{n=1}^{\infty}\left|G\left(2^{n}, \alpha_{n}\right)\right|=\infty
$$

По лемме Кальдерона (см., например, [1, гл. 3, лемма 3.3]) подберем сдвиги $\left\{\tau_{n}\right\}_{n} \geqslant 1$ так, чтобы

$$
\left|\limsup _{n} \tau_{n}\left\{G\left(2^{n}, \alpha_{n}\right)\right\}\right|=1 .
$$

Положим теперь

$$
f=\sum_{n=1}^{\infty} \chi_{\tau_{n}\left\{E\left(2^{n}, \alpha_{n}\right)\right\}}
$$

Тогда

$$
\|f\|_{p} \leqslant \sum_{n=1}^{\infty}\left\|\chi_{\tau_{n}\left\{E\left(2^{n}, \alpha_{n}\right)\right\}}\right\|_{p}=\sum_{n=1}^{\infty}\left(2^{n} \alpha_{n}^{2}\right)^{1 / p}=\sum_{n=1}^{\infty}\left(2^{n} n\right)^{-1 / p}<\infty .
$$


Таким образом, $f \in L^{p}\left(I^{2}\right)$, и так как

$$
\sum_{n=1}^{\infty}\left|E\left(2^{n} \alpha_{n}\right)\right|=\sum_{n=1}^{\infty}\left(2^{n} n\right)^{-1}<\frac{1}{2}
$$

то мера тех точек, где $f>0$, не превосходит $1 / 2$. Стало быть, существует множество $\mathscr{P} \subset I^{2},|\mathscr{P}| \geqslant 1 / 2$, где $f(x)=0$. С другой стороны, каждая точка $x \in \mathscr{P}$ принадлежит некоторому $\tau_{n}\left\{G\left(2^{n}, \alpha_{n}\right)\right\}$ со сколь угодно большим номером $n$. Но тогда для параллелограмма $\widehat{R}_{\nu}^{n} \ni x$ справедливо (19). Следовательно, для $x \in \mathscr{P}$ и выпуклых $R$

$$
\limsup _{\operatorname{diam}(R) \rightarrow 0}|R|^{-1} \int_{R \ni x} f(y) d y \geqslant \frac{1}{8}>f(x)=0,
$$

что и дает требуемую недифференцируемость интеграла от $f$.

Оценим теперь гладкость функции $f$. Обозначив через $\chi_{n}(x)$ характеристическую функцию множества $E\left(2^{n}, \alpha_{n}\right)$, получим для частного модуля по второй переменной следуюшую оценку

$$
\omega_{2}\left(\chi_{n} ; h\right)_{p} \leqslant\left(\sum_{i=1}^{2^{n}} \sum_{j=1}^{2^{i-1}} \int\left|\Delta_{h}^{(2)} \chi_{\Pi\left(i, j, \alpha_{n}\right)}(x)\right|^{p} d x\right)^{1 / p} .
$$

Оценим теперь интеграл. Обозначив через $\Pi_{h}^{(2)}\left(i, j, \alpha_{n}\right)$ результат сдвига $\Pi\left(i, j, \alpha_{n}\right)$ вдоль второй оси на величину $h$, а через $\Delta-$ симметрическую разность множеств, получим

$$
\int\left|\Delta_{h}^{(2)} \chi_{\Pi\left(i, j, \alpha_{n}\right)}(x)\right|^{p} d x=\left|\Pi\left(i, j, \alpha_{n}\right) \Delta \Pi_{h}^{(2)}\left(i, j, \alpha_{n}\right)\right| .
$$

Выясним, при каких сдвигах $\left(i, j, \alpha_{n}\right) \cap \Pi_{h}^{(2)}\left(i, j, \alpha_{n}\right) \neq \varnothing$.

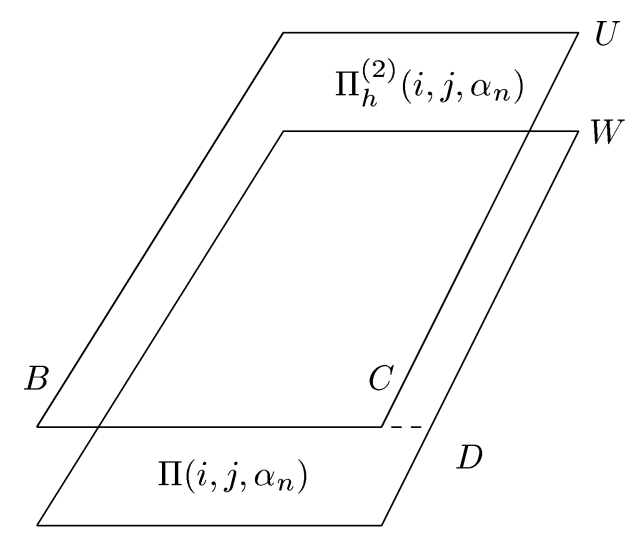

A

E

Рис. 8 
На рис. $8 h=|C E|$. Поскольку угол $A$ не менее чем $\pi / 4$, то при условии $h \leqslant$ $|A E|$ как раз и получится требуемое расположение параллелограммов $\Pi\left(i, j, \alpha_{n}\right)$ и $\Pi_{h}^{(2)}\left(i, j, \alpha_{n}\right)$. Однако $|A E|=\alpha_{n} 2^{-i}$, а так как $1 \leqslant i \leqslant 2^{n}$, то достаточно, чтобы $h \leqslant \alpha_{n} 2^{-2^{n}}$. Тогда

$$
\begin{aligned}
\left|\Pi\left(i, j, \alpha_{n}\right) \Delta \Pi_{h}^{(2)}\left(i, j, \alpha_{n}\right)\right| & =2(|A B C D|+|C D W U|) \\
& =2\left(h \alpha_{n} 2^{-i}+\left(\alpha_{n}-h\right)|C D|\right) .
\end{aligned}
$$

Далее, $|C D| \leqslant|C E|=h$, и, таким образом,

$$
\left|\Pi\left(i, j, \alpha_{n}\right) \Delta \Pi_{h}^{(2)}\left(i, j, \alpha_{n}\right)\right| \leqslant 4 \alpha_{n} h .
$$

Следовательно, в этом случае

$$
\begin{aligned}
\omega_{2}\left(\chi_{n} ; h\right)_{p} & \leqslant\left(\sum_{i=1}^{2^{n}} \sum_{j=1}^{2^{i-1}} 4 \alpha_{n} h\right)^{1 / p} \\
& =\left(\sum_{i=1}^{2^{n}} 2^{i} 2^{-n} n^{-1 / 2} h\right)^{1 / p} \leqslant h^{1 / p}\left(2^{2^{n}} 2^{-n} n^{-1 / 2}\right)^{1 / p}
\end{aligned}
$$

Пусть теперь $\alpha_{n} 2^{-2^{n}} \leqslant h<\alpha_{n-1} 2^{-2^{n-1}}$. Тогда

$$
\begin{aligned}
\omega_{2}(f ; h)_{p} & \leqslant \sum_{k=1}^{n-1} \omega_{2}\left(\chi_{k} ; h\right)_{p}+\sum_{k=n}^{\infty}\left\|\chi_{k}\right\|_{p} \\
& \leqslant h^{1 / p} \sum_{k=1}^{n-1}\left(2^{2^{k}} \alpha_{k}\right)^{1 / p}+\sum_{k=n}^{\infty}\left(2^{k} \alpha_{k}^{2}\right)^{1 / p} \\
& \leqslant h^{1 / p}\left(2^{2^{n-1}} \alpha_{n-1}\right)^{1 / p}+2 \sum_{k=n}^{\infty}\left(2^{-k} k^{-1}\right)^{1 / p} \\
& \leqslant\left(2^{-2^{n-1}} \alpha_{n-1}\right)^{1 / p}\left(2^{2^{n-1}} \alpha_{n-1}\right)^{1 / p}+\frac{2}{\left(2^{n} n\right)^{1 / p}} \\
& =\left(\alpha_{n-1}\right)^{2 / p}+\frac{2}{\left(2^{n} n\right)^{1 / p}} \\
& =\frac{1}{\left(2^{n} \sqrt{n}\right)^{2 / p}}+\frac{2}{\left(2^{n} n\right)^{1 / p}} \leqslant \frac{3}{\left(2^{n} n\right)^{1 / p}} .
\end{aligned}
$$

Поскольку $\log (1 / h) \asymp 2^{n}$, то

$$
\omega_{2}(f ; h)_{p}=O\left\{\log \frac{1}{h} \log \log \frac{1}{h}\right\}^{-1 / p}
$$

и, таким образом, осталось оценить частньй модуль непрерывности по первой переменной. 
Как и вьше, обозначим через $\Pi_{h}^{(1)}\left(i, j, \alpha_{n}\right)$ результат сдвига $\Pi\left(i, j, \alpha_{n}\right)$ вдоль первой переменной на величину $h$. Из геометрических рассмотрений ясно, что для $\alpha_{n} 2^{-2^{n}} \leqslant h \leqslant \alpha_{n-1} 2^{-2^{n-1}}$ и для $1 \leqslant k \leqslant n$ длина основания П $\left(i, j, \alpha_{k}\right)$ равна $\alpha_{k} 2^{-i} \geqslant h$, а потому $\left(i, j, \alpha_{k}\right) \cap \Pi_{h}^{(1)}\left(i, j, \alpha_{k}\right) \neq \varnothing$. Тогда

$$
\begin{aligned}
\omega_{1}\left(\chi_{k} ; h\right)_{p} & \leqslant\left(\sum_{i=1}^{2^{k}} \sum_{j=1}^{2^{i-1}} \int\left|\Delta_{h}^{(1)} \chi_{\Pi\left(i, j, \alpha_{k}\right)}(x)\right|^{p} d x\right)^{1 / p} \\
& \leqslant\left(\sum_{i=1}^{2^{k}} \sum_{j=1}^{2^{i-1}}\left|\Pi\left(i, j, \alpha_{k}\right) \cap \Pi_{h}^{(1)}\left(i, j, \alpha_{k}\right)\right|\right)^{1 / p} \\
& \leqslant\left(\sum_{i=1}^{2^{k}} \sum_{j=1}^{2^{i-1}} 2 h \alpha_{k}\right)^{1 / p} \ll\left(h \alpha_{k} 2^{2^{k}}\right)^{1 / p} .
\end{aligned}
$$

Следовательно,

$$
\omega_{1}(f ; h)_{p} \leqslant \sum_{k=1}^{n-1} \omega_{1}\left(\chi_{k} ; h\right)_{p}+\sum_{k=n}^{\infty}\left\|\chi_{k}\right\|_{p} \leqslant h^{1 / p} \sum_{k=1}^{n-1}\left(2^{2^{k}} \alpha_{k}\right)^{1 / p}+\sum_{k=n}^{\infty}\left(2^{k} \alpha_{k}^{2}\right)^{1 / p},
$$

а эта сумма уже рассматривалась при оценке частного модуля непрерывности по второй переменной (см. (20)). Повторяя дословно упомянутые рассуждения, получим требуемую оценку для частного модуля непрерывности по первой переменной.

Таким образом, теорема 6 доказана.

\section{§ 6. Некоторые замечания и следствия}

СлеДСтвИЕ. Интегралы функций из пространств $B_{p, 1}^{(d-1) / p}\left(I^{d}\right)$ дифференчируются базисом из выпуклых множеств.

Поскольку базис из выпуклых множеств не является плотностным, то привлечение информации о гладкости функции является сушественным моментом, тем более, что $B_{p, 1}^{(d-1) / p}\left(I^{d}\right) \not \subset L^{\infty}\left(I^{d}\right), d \geqslant 2$.

Отметим, что вопросы дифференцирования интегралов в близкой шкале классов Лизоркина-Трибеля $F_{p, q}^{s}\left(I^{d}\right)$ остаются открытыми.

Сделаем теперь несколько замечаний.

1. Интересно было бы выяснить, является ли необходимьм множитель $\psi(\operatorname{diam}(P))$ в оценке скорости дифференцирования (8). Аналогичную оценку можно получить и для классов Соболева $W_{p}^{1}$, причем, в случае $p>1$ (когда $\left.W_{p}^{1}=\operatorname{Lip}(1, p)\right)$ упомянутый множитель можно опустить. Тем не менее, можно ли его опустить для класса $W_{1}^{1}$ нам не известно.

2. Заметим, что мы доказали несколько более сильное утверждение, чем теорема 3 , так как при доказательстве использовалась только информация о втором частном модуле непрерывности функции $f$. Учитывая инвариантность базиса из вьпуклых множеств относительно поворотов, приходим к вьводу, что для дифференцируемости интеграла от функции базисом из выпуклых множеств достаточно 
иметь наилучшую гладкость в некотором направлении, что никак не ограничивает глобальный рост функции.

3. Как уже отмечалось, А. Зигмунд доказал недифференцируемость класса характеристических функций измеримых множеств базисом из произвольно ориентируемых прямоугольников. Вместе с тем, он так же установил дифференцируемость этим базисом класса характеристических функций открытых и замкнутых множеств. Насколько нам известно, другие классы множеств более не рассматривались.

Наша теорема 2 позволяет ввести еще один класс множеств. А именно, это класс множеств конечного периметра в смысле Де Джоржи и Каччипполи (см. [21]). Обозначим этот периметр множества $E$ через $\pi(E)$. Так как [22, с. 238]

$$
\pi(E) \asymp \sup _{h>0} \frac{\omega\left(\chi_{E} ; h\right)_{1}}{h}
$$

то дифференцируемость интегралов от характеристических функций этих множеств есть прямое следствие теоремы 2.

4. Как мы установили, базис $\mathscr{B} 0$ с "неравномерными сечениями", и базис из гибких цилиндров $\mathscr{B}$ с “равномерньми сечениями”, отличаются в шкале Липшицевых классов на порядок показателя $1 / p$. Интересно было бы ввести характеристику степени "равномерности" сечений, и с помощью этой характеристики определить базисы, дифференцирующие в точности промежуточные классы $\operatorname{Lip}(\alpha, p)$, $(d-1) / p<\alpha<d / p$.

5. Результаты $\S 5$ частично анонсированы нами в [23].

\section{Список литературы}

1. Гусман M. Дифференцирование интегралов в $\mathbb{R}^{n}$. М.: Наука, 1978.

2. de Guzman M. Real Variable Methods in Fourier Analysis. V. 46. Amsterdam: North-Holland Math. Stud., 1981.

3. Busemann H., Feller W. Zur Differentiation der Lebesgueschen Integrale // Fund. Math. 1934. V. 22. P. 226-256.

4. Lebesgue A. Surl'integration des fonctions discontinues // Ann. Sci. Ecole Norm. Sup. (4). 1910. V. 27. P. 361-450.

5. Сакс С. Теория интеграла. М.: ИЛ, 1949.

6. Strömberg J. O. Weak estimates on maximal functions with rectangles in certain directions // Ark. Math. 1977. V. 15. P. 229-240.

7. Sjögren P., Sjölin P. Littlewood-Paley decompositions and Fourier multipliers with singularities on certain sets // Ann. Inst. Fourier (Grenoble). 1981. V. 31. P. 157-175.

8. Nikodym $O$. Sur la mesure des esembles plans dont tous les points sonts rectilinearement accessibles // Fund. Math. 1927. V. 10. P. 116-168.

9. Ульянов П. Л. Об абсолютной и равномерной сходимости рядов Фурье // Матем. сб. 1967. Т. 72 (114). С. $193-224$.

10. Темиргалиев $H$. Об абсолютной и равномерной сходимости кратных рядов Фурье // Матем. заметки. 1972. Т. 12. №2. С. 139-148.

11. Коляда В. И. О вложении непрерывных функций многих переменных // Матем. сб. 1976. T. 99 (141). № 3. С. 421-432.

12. Стоколос А. М. О сильном дифференцировании интегралов функций из классов Гёльдера // Матем. заметки. 1994. Т. 55. № 1. С. 84-104. 
13. Stein E. M., Wainger S. Problems in Harmonic Analysis related to curvature // Bull. Amer. Math. Soc. 1978. V. 84. P. 1239-1295.

14. Blozinsky B. Multivariate rearrangements and Banach function spaces with mixed norms // Trans. Amer. Math. Soc. 1981. V. 263. №1. P. 149-167.

15. Стороженко Э. А. Необходимые и достаточные условия для вложения некоторых классов функций // Изв. АН СССР. Сер. матем. 1973. Т. 39. С. 386-398.

16. Коляда В. И. Оценки перестановок и теоремы вложения // Матем. сб. 1988. Т. 136. № 1. C. 3-23.

17. Коляда В. И. Перестановки функций и теоремы вложения // УМН.. Т. 44. № 5. С. 61-95.

18. Никольский C. М. Приближение функций многих переменных и теоремы вложения. М.: Наука, 1969.

19. Стейн E. М. Сингулярные интегралы и дифференциальные свойства функций. М.: Мир, 1973.

20. Хёрмандер Л. Анализ линейных дифференциальных операторов с частньпи производными. Т. 1 (Теория распределений и анализ Фурье). М.: Мир, 1986.

21. Джсусти Е. Минимальные поверхности и функции ограниченной вариации. М.: Мир, 1989.

22. Мазья В. Г., Шапошникова Т. О. Мультипликаторы в пространствах дифференцируемых функций. Ленинград: Изд-во ЛГУ, 1986.

23. Стоколос A. М. О дифференцировании интегралов базисами из выпуклых множеств // Международная конференция "Функциональные пространства, теория приближений, нелинейньй анализ” (апрель-май 1995 г.). Тезисы докладов. М.: Изд-во ПАИМС, 1995. C. 260-261.

Одесский государственњщй университет

Институт Математики, Экономики и Механики

E-mail: oleg@leon.odessa.ua
Поступила в редакцию 03.10 .1995 